\title{
Two Core Promotor Mutations Identified in a Hepatitis B Virus Strain Associated with Fulminant Hepatitis Result in Enhanced Viral Replication
}

\author{
Thomas F. Baumert, ${ }^{\star \ddagger}$ Steven A. Rogers, ${ }^{\star}$ Kiyoshi Hasegawa, ${ }^{\star}$ and T. Jake Liang ${ }^{\star \ddagger}$ \\ * Gastrointestinal Unit, Department of Medicine, Massachusetts General Hospital and Harvard Medical School, Boston, Massachusetts \\ 02114; and $\ddagger$ Liver Diseases Section, National Institute of Diabetes and Digestive and Kidney Diseases, National Institutes of Health, \\ Bethesda, Maryland 20892
}

\begin{abstract}
Viral mutations have been implicated in alteration of the biological phenotype of hepatitis B virus (HBV). We recently cloned and sequenced the viral genome of an HBV strain associated with an outbreak of fulminant hepatitis (FH strain). The FH strain contained numerous mutations in all genomic regions and was functionally characterized by a more efficient encapsidation of pregenomic RNA leading to highly enhanced replication. To define the responsible mutation(s) for the enhanced replication, we introduced individual mutations of the FH strain into a wild-type construct by oligonucleotide-directed mutagenesis. Analysis of viral replication showed that two adjacent mutations in the $\mathrm{HBV}$ core promotor ( $\mathrm{C}$ to $\mathrm{T}$ at nucleotide 1768 and $\mathrm{T}$ to $\mathrm{A}$ at nucleotide 1770) led to high level replication. Similar to the FH strain, this mutant displayed the phenotype of enhanced encapsidation of pregenomic RNA. Functional studies in an encapsidation assay demonstrated that the identified mutations resulted in a minor increase of pregenomic RNA transcription (two- to threefold) and a major transcription-independent enhancement ( $>10$-fold) of viral encapsidation. Our results demonstrate that the two adjacent mutations in the HBV core promotor region are responsible for the enhanced replication of the FH strain. These two mutations, outside the previously described encapsidation signal, core, and polymerase polypeptides, appeared to affect a novel genetic element involved in viral encapsidation. (J. Clin. Invest. 1996. 98:2268-2276.) Key words: core promotor • encapsidation $\cdot \mathrm{HBX}$ gene
\end{abstract}

\section{Introduction}

Hepatitis B virus $(\mathrm{HBV})^{1}$ is a partially double-stranded DNA virus that replicates through an RNA intermediate (for review, see references 1 and 2). The virally encoded reverse transcriptase-polymerase (RT-Pol) is essential for this unique form

This work was presented in part at the annual meeting of the Molecular Biology of Hepatitis B Virus, 23-27 July 1995, San Diego, CA, and at the 46th Annual Meeting of the American Association for the Study of Liver Diseases, 3-7 November 1995, Chicago, IL.

Address correspondence to T.J. Liang, Liver Diseases Section, National Institute of Diabetes and Digestive and Kidney Diseases, National Institutes of Health, Building 10-9B16, 10 Center Drive, Bethesda, MD 20892-1800. Phone: 301-496-1721; FAX: 301-402-0491; E-mail: jliang@nih.gov

Received for publication 18 June 1996 and accepted in revised form 13 September 1996.

The Journal of Clinical Investigation

Volume 98, Number 10, November, 1996, 2268-2276 of genome replication. Viral replication occurs exclusively in the core particle which is assembled through complex interactions among pregenomic RNA, core protein, and RT-Pol in a process called encapsidation. The capsid containing the replicative intermediate is then enveloped by three forms of HBV surface antigens (HBsAg) in a lipid bilayer. Although the molecular mechanism of HBV replication has been largely elucidated with the identification of various essential elements (3-8), it is not clear whether any other sequences outside these elements play an important role in the replicative process.

We have previously described the association of an HBV variant with an outbreak of fulminant hepatitis (FH) B (9). This nosocomial outbreak of five cases was remarkable for an unusually severe course of acute HBV infection, leading to $100 \%$ mortality within a few days from hepatic and renal failure. The HBV strain (FH strain) responsible for this outbreak contained two $\mathrm{G}$ to $\mathrm{A}$ mutations in the precore open reading frame, one of them resulting in a stop codon (9). Cloning and sequencing of the entire genome of this viral strain revealed numerous mutations affecting conserved and nonconserved sequences in various regions of the HBV genome (10). Functional characterization of the FH strain in transfected hepatoma cells showed a highly increased viral replication (10). Enhanced replication of the FH strain was caused by a more efficient encapsidation of pregenomic RNA into the viral core particles. Our previous study demonstrated that the precore mutations were not responsible for enhanced replication (10). In the woodchuck model, the precore stop codon mutation had no effect on viral replication and the initial course of infection, but appeared to result in less chronic infection (11). These observations indicated that other mutation(s) in the HBV genome may be important for the altered biological behavior of the FH strain. In this study, we identified and characterized functionally the mutations responsible for the enhanced replication of this HBV strain.

\section{Methods}

Constructs. Replication-competent constructs of wild-type (adwR9, aywR9) and fulminant HBV (FHR9) strains were described previously (10). These constructs contained 1.2 times the genomic length of HBV. The HBX expression constructs HBXadw and HBXFH were obtained by subcloning the HpaI-BglII fragment of adwR9 and FHR9, respectively, into SmaI and BamHI sites of pGEM7Z(+). HBXMT5/6 was generated by ligating the XhoI-SacII fragment of

1. Abbreviations used in this paper: $\mathrm{ccc}$, covalently closed circular; $\mathrm{FH}$, fulminant hepatitis; $\mathrm{HBeAg}$, hepatitis B virus e antigen; $\mathrm{HBsAg}$, hepatitis $B$ virus surface antigen; $\mathrm{HBV}$, hepatitis B virus; $\mathrm{HBXKO}$, HBX knockout; hGH, human growth hormone; MT, mutant; nt, nucleotide; ORF, open reading frame; RT-Pol, reverse transcriptase-polymerase; WT, wild-type. 
HBXadw and the SacII-BglII fragment of pSelectHBV mutant (MT)5/6 (described below) into the XhoI and BamHI sites of pGEM7Z $(+)$. The core promotor constructs pXp2CPadw and pXp2CPMT5/6 were generated by digesting pHBXadw and HBXMT5/6 with AflIII (HBV nucleotide [nt] 1852), filling in the ends with Klenow, and then digesting with XhoI present in the $5^{\prime}$ end of the multiple cloning site of pGEM7Z $(+)$. The fragment was then cloned into the SalI and SmaI sites of $\mathrm{pXp} 2$ which contains a promotorless luciferase gene $(12,13)$. These constructs contained the luciferase gene driven by wild-type and mutant HBV core promotors including HBV sequences up to enhancer I (nt 965-1852). pCPadwlacZ was constructed by ligating the HincII (nt 1684)-AflIII (nt 1852) fragment of pHBXadw, the AflIII-KpnI fragment of pE-lacZ (14) and the SmaIKpnI fragment of pE-lacZ. pCPMT5/6lacZ was similarly constructed using pHBXMT5/6. In these two constructs, the CMV promotor of pE-lacZ was replaced with the wild-type or mutant HBV core promotor. All mutant constructs were confirmed by DNA sequencing. Covalently closed circular DNA was generated from wild-type (WT) (adwR9) or mutant (MT5/6R9) HBV-DNA as described previously (15).

Oligonucleotide mutagenesis. The Altered Sites In Vitro System (Promega Corp., Madison, WI) was used to introduce HBV core promotor, precore, core and HBX knockout (HBXKO) mutations: the first mutation with $\mathrm{T}$ to $\mathrm{G}$ at nucleotide 1848 (MT2), the second with a $\mathrm{G}$ to $\mathrm{T}$ and $\mathrm{T}$ to $\mathrm{A}$ at nucleotides 1754 and 1756 (MT 3/4), the third with a $\mathrm{C}$ to $\mathrm{T}$ and $\mathrm{T}$ to $\mathrm{A}$ at nucleotides 1768 and 1770 (MT5/6), the fourth with $\mathrm{C}$ to $\mathrm{T}$ at nucleotide 1768 (MT5), and the fifth with $\mathrm{T}$ to $\mathrm{A}$ at nucleotide 1770 (MT6). The five mutant oligonucleotides were: 5' CTAATCATCTCGTGTACATGTC $3^{\prime}$ (sense, nt 1837-1859) for MT2, 5' GGGGGAGGATAATAGGTTAAAGGTC 3' (sense, nt 1744-1768) for MT3/4, 5' AGGTTAAAGGTTTATGTATTAGGAG 3' (sense, nt 1757-1781) for MT5/6, 5' AGGTTAAAGGTTTTTGTATTAGGAG 3' (sense, nt 1757-1781) for MT5, and 5' AGGTTAAAGGTCTÄTGTATTAGGAG 3' (sense, nt 1757-1781) for MT6. To generate HBXKO mutations the following primers were used: 5' TGAACGCCCATTAGATCCTGCCCAA 3' (to generate a stop codon for the HBX.2 protein p8) and 5' ACTCCCAGCAAGGTCAACGACCGA 3' (to generate a mutated start codon for HBX.3 protein p6.6). Wild-type HBVadw DNA was used as template for mutagenesis (pSelectHBVadw). These two mutations were designed to minimize effects on other open reading frames, such as reverse transcriptase, polymerase, or on overlapping conserved cis-acting sequences, such as enhancer and promotor sequences. The replication-competent mutant constructs were generated by cloning the RsrII-BglII fragment (nt 1574-1986) of pSelectHBVMutant into adwR9. The HBXKO constructs were generated by cloning the SacII-StuI fragment (nt 1474-1704) of pSelectHBXKO into adwR9 or MT5/6R9.

MT7, containing a change of $\mathrm{T}$ to $\mathrm{C}$ at nucleotide 1982 in the core coding sequence, was generated by PCR mutagenesis using a primer containing the endogenous BglII site of HBV (nt 1986). The following primers were used to generate the mutant DNA fragment: 5' CGGAGCTCCTAGCCGCTTGTTTTGCTCGCAGC 3' (sense, nt 12811307) and 5' TAGAAGATCTCGTGCGGAAGGAAAGAA 3' (antisense, nt 1969-1995) for MT7. After subcloning the PCR-generated mutant fragment into PCR II vector (Invitrogen, San Diego, CA), an RsrII-BglII fragment was subcloned into the replication-competent construct adwR9 in the same way as described above. All mutant constructs were sequenced to confirm the correct mutations and ensure absence of other mutations that could have been potentially introduced during mutagenesis.

Tissue culture and transfection. Human hepatoma HuH-7 and HepG2 cells were maintained in DME (Gibco, Grand Island, NY) plus $10 \%$ FBS (Sigma Chemical Co., St. Louis, MO) at $37^{\circ} \mathrm{C}$ and $5 \%$ $\mathrm{CO}_{2}$. HuH-7 or HepG2 cells were grown to $70 \%$ confluence and transfected with DNA by a $\mathrm{CaPO}_{4}$ transfection kit (Calcium Phosphate Mammalian Cell Transfection kit; 5 Prime-3 Prime Inc., Boulder, $\mathrm{CO})$.
Analysis of transfection efficiency. The pTKGH plasmid containing the human growth hormone (hGH gene driven by the thymidine kinase enhancer and promotor) was cotransfected with various HBV constructs to control for transfection efficiency. Typically, $15 \mu \mathrm{g}$ of HBV construct was cotransfected with $1 \mu \mathrm{g}$ of pTKGH plasmid into $\mathrm{HuH}-7$ cells grown in 10-cm dishes. From each transfection experiment, medium was harvested and hGH was measured with a radioimmunoassay from Nichols Institute Diagnostics (San Juan Capistrano, CA). A typical result for hGH is shown in Table I.

Viral protein, $R N A$, and $D N A$ analyses. $\mathrm{HBsAg}$ and $\mathrm{HBe} \mathrm{Ag}$ were determined by commercially available radioimmunoassays (Abbott Laboratories, North Chicago, IL). 3 or $4 \mathrm{~d}$ after transfection, HuH-7 cells were harvested for viral RNA and DNA analysis. RNA was prepared by the guanidium isothiocyanate-acid-phenol method (16) analyzed by formaldehyde agarose gel electrophoresis $(10 \mu \mathrm{g}$ RNA) and hybridized with an HBV-specific probe. Viral replicative intermediates associated with intracellular core particles were isolated by ultracentrifugation of cell lysate through a $30 \%$ sucrose cushion and then analyzed by Southern blot hybridization (17).

Analysis of core-associated RNA. $3 \mathrm{~d}$ after transfection of HuH-7 cells with various HBV constructs, core particles were purified by ultracentrifugation as described above. The pellet containing the core particles was treated with Staphylococcus aureus nuclease (Boehringer Mannheim, Indianapolis, IN) at a concentration of $8 \mu \mathrm{g} / \mathrm{ml}$ for $60 \mathrm{~min}$ at $37^{\circ} \mathrm{C}$ to eliminate any contaminating nucleic acids. Coreassociated RNA was then isolated by the guanidium isothiocyanateacid-phenol method twice (16). For primer extension analysis, an HBV primer (5' TCTAAGGCTTCTCGATACAGAGCTG $3^{\prime}$ ) spanning nt 2006-2030 in the antisense orientation was end labeled with $\gamma-\left[{ }^{32} \mathrm{P}\right] \mathrm{ATP}$ and then reacted with RNA by a standard protocol (18). Core-associated RNA was compared with total RNA prepared as above. In addition, core-associated RNA was analyzed by RNAse-protection analysis (RPA II kit; Ambion Inc., Austin, TX). An $\alpha-\left[{ }^{32} \mathrm{P}\right] \mathrm{CTP}-$ labeled HBV-RNA probe (AvaI-BglII fragment of HBVadw) was generated by in vitro transcription of the linearized (digested with AvaI) plasmid pH BVadwBH (In Vitro Transcription kit; Promega Corp.). pHBVadwBH was constructed by subcloning an AatII (nt 1417)-BglII (nt 1986) fragment of HBVadw into pGEM7 (AatII and BamHI sites). Primer extension products and protected RNA fragments were analyzed on an $8 \%$ polyacrylamide-urea gel.

Encapsidation assay. Cis and trans effects of MT5/6 on encapsidation of pregenomic RNA were studied using an encapsidation assay described previously $(14,19)$. Plasmid pE-LacZ (a generous gift from Don Ganem) contains a fusion of the HBV encapsidation signal sequence (nt 1815-1986) and the lacZ gene driven by the CMV promotor. Transcription from the CMV immediate-early promotor initiates at nt 1815 of the HBV sequence and leads to a chimeric HBV $\epsilon-$ lacZ RNA. In plasmids pCPadwlacZ and pCPMT5/6lacZ, transcription of the chimeric RNA is driven by the HBV core promotor (wild-type and mutant). By providing core and polymerase proteins in trans, the chimeric HBV $\epsilon$-lacZ RNA has been shown to be encapsidated into core particles in tissue culture (14). HBV $\epsilon$-lacZ RNA was analyzed by ribonuclease protection assay as described (5). Probe lacZ, which was generated by in vitro transcription of pLacZ (a generous gift from Don Ganem, University of California, San Francisco, CA) is a 475-nt riboprobe with 50 polylinker nucleotides and $425 \mathrm{nt}$ complementary to the lacZ sequence of the HBV $\epsilon$-lacZ RNA (5).

Analysis of core promotor activity and transactivation function of $H B X$. Core promotor activities were studied by analyzing luciferase gene expression of the plasmids pXp2CPadw and pXp2CPMT5/6 in transiently transfected HepG2 cells $(0.5 \mu \mathrm{g}$ DNA per six-well plate). The plasmids $\mathrm{pXp} 2$ (promotorless) and RSVLuc (containing the luciferase gene driven by the RSV promotor $(12,13)$ served as negative and positive controls. Luciferase activities were determined using the Luciferase reagent (Promega Corp.) with a luminometer. For analysis of HBX transactivation, $0.1 \mu \mathrm{g}$ of HBXadw or HBXFH was cotransfected with $0.5 \mu \mathrm{g}$ DNA of RSVLuc into triplicate of HepG2 cells maintained in six-well plates. 
Figure 1. Mapping of the HBV sequences responsible for enhanced replication. Two regions of adw strain, RsRII to XhoI $(R X)$, RsRII to BglII $(R B g)$, were replaced with $\underline{\mathrm{kb}}$

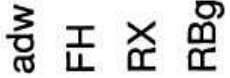

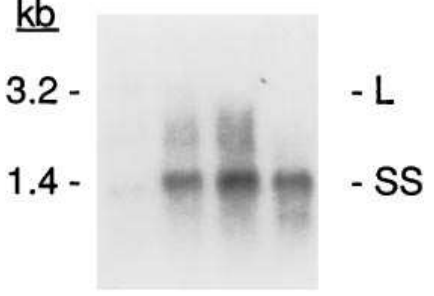

12334 equivalent regions of the $\mathrm{FH}$ strain in a replication-competent construct (adwR9). HuH-7 cells were transfected with the chimeric constructs and core particle-associated HBV DNAs were isolated and subjected to Southern blot analysis. Lane contents are as indicated.

\section{Results}

Identification of mutation(s) responsible for enhanced replication. To further define the HBV sequences responsible for enhanced replication of FH strain, various regions of wild-type strain were replaced with equivalent regions of the $\mathrm{FH}$ strain in the replication competent construct adwR9. The chimeric constructs were analyzed for viral replication after transfection into HuH-7 cells (Fig. 1). The responsible region of the fulminant strain was mapped to a fragment spanning from nucleotide 1574 to 1986 (RsrII-BgIII). This region contains nine mutations affecting conserved nucleotide sequences (10). We selected six of these mutations and introduced them individually or in combination into the wild-type adwR9 construct (MT2-7, Fig. 2). These mutations of the FH strain were located in the HBX gene, the core promotor, the encapsidation signal, and the core protein. These constructs were transfected into $\mathrm{HuH}-7$ cells and viral replication was analyzed (Fig. 3). Only the construct MT5/6 demonstrated the phenotype of enhanced replication similar to the $\mathrm{FH}$ strain, while the other mutations had no effect. Densitometric quantification revealed an $\sim 17$ fold (17.5 \pm 7.7 , from four experiments) difference between wild-type and mutant (MT5/6) signal intensities for the replica-

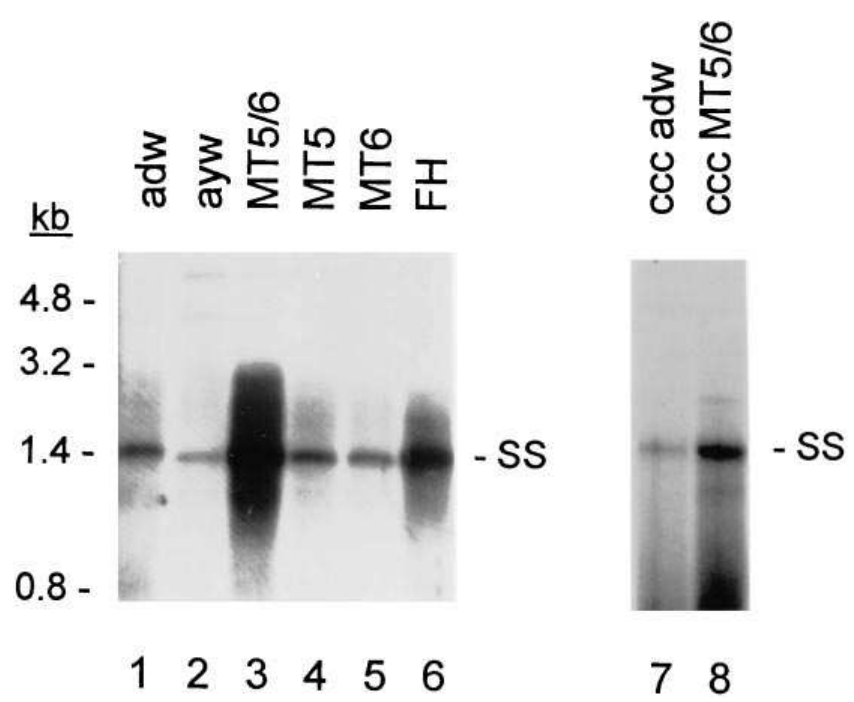

Figure 3. HBV replication in cells transfected with HBV constructs and ccc HBV-DNA. $4 \mathrm{~d}$ after transfection, HuH-7 cells were harvested and core particle-associated HBV DNAs were isolated and subjected to Southern analysis. Lane contents are as indicated. SS, single-stranded HBV DNA. Results are representative of four independent experiments.

tive intermediates. The actual difference may be much greater because densitometry of autoradiography is only linear over one log. To examine whether both mutations 5 and 6 are required for the enhanced replication, constructs containing the individual mutations 5 and 6 (MT5 and MT6) were generated and analyzed for replication (Fig. 3). Mutants 5 or 6 by itself appeared to have only a minor nonsignificant effect on replication. Densitometric analysis from four experiments showed a $1.47 \pm 0.63$ - and $1.49 \pm 0.87$-fold increase of replicative intermediates over the wild type for MT5 and MT6, respectively. To confirm the effect of MT5/6 on HBV replication in a more physiological setting, we generated covalently closed circular (ccc) DNA from wild-type and MT5/6 genome to better corre-

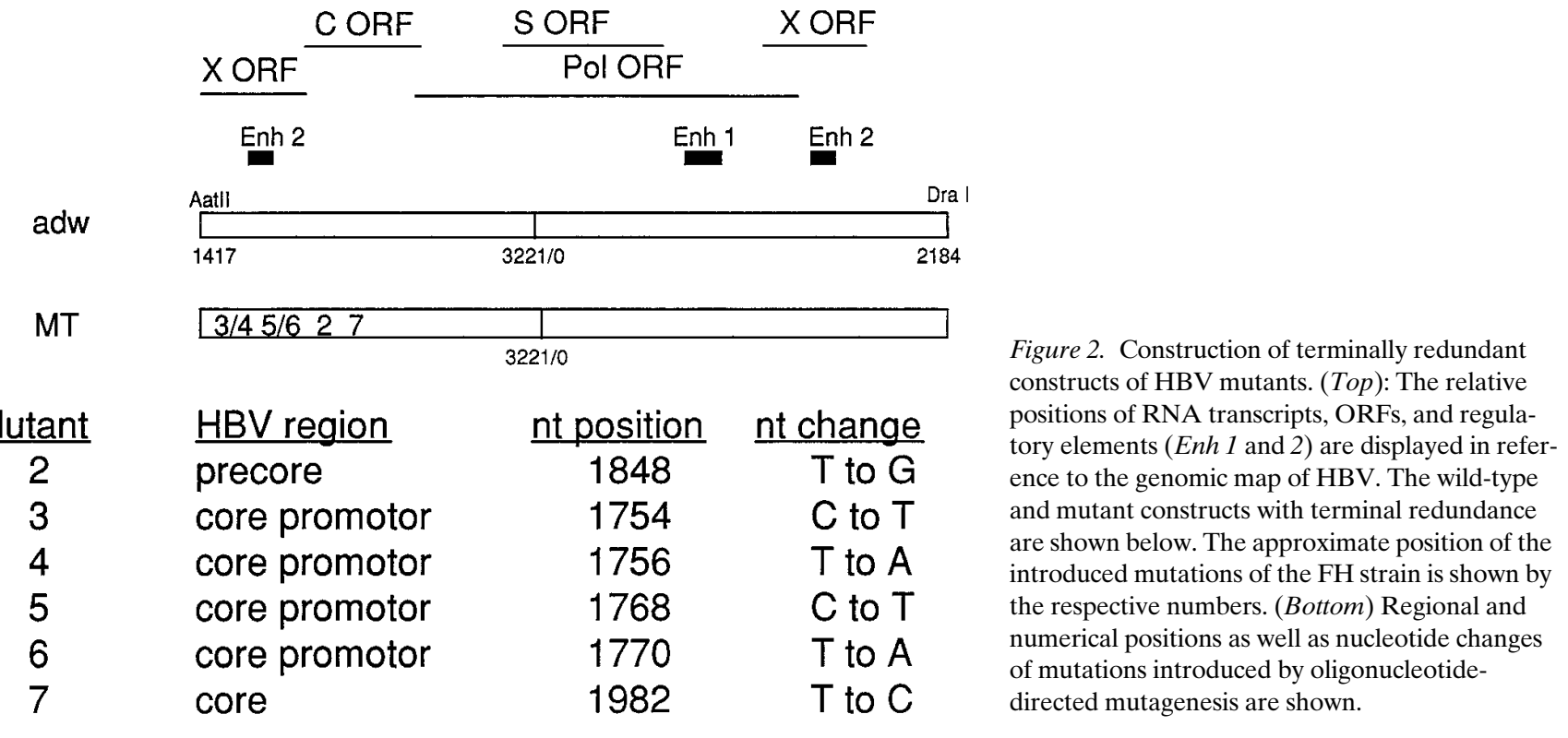


spond to the natural template for the transcription of viral mRNAs. Analysis of viral replication of ccc HBV-DNA demonstrated a similar effect of MT5/6 on HBV replication as shown with the terminal-redundant R9 construct (Fig. 3). The two identified mutations (nt 1768 and 1770) are within the overlapping region of the HBV core promotor and the HBX gene.

Enhanced replication results from enhanced encapsidation of pregenomic RNA. To define the molecular mechanism of enhanced viral replication, we studied the functional effect of MT5/6 on viral encapsidation, transcription, protein expression, and function. Since the fulminant strain was characterized by a more efficient encapsidation of pregenomic RNA, we first analyzed the level of viral encapsidation of the mutant constructs in comparison to the wild-type strains (Fig. 4). Only construct MT5/6 showed a highly increased encapsidation of pregenomic RNA (Fig. 4). Densitometric analysis revealed that MT5/6 showed an $\sim 12$-fold (12.1 \pm 4.4 , from four experiments) increase in the signal intensitiy of encapsidated pregenomic RNA compared to the wild type. In contrast, the constructs containing the individual mutations MT5 or MT6 displayed only a minimal increase of encapsidation (MT5: 2.06 \pm 0.86 -fold; MT6: 1.94 \pm 0.72 -fold; from four experiments) compared with the wild-type constructs (Fig. 4). This finding was confirmed independently by ribonuclease protection analysis of encapsidated RNA (not shown).

The identified mutations affect core promotor activity leading to increased pregenomic RNA transcription. Since these two mutations were located within the core promotor, they might enhance transcription of pregenomic RNA resulting in the apparent increased encapsidation. Total viral RNAs from cells transfected with these constructs were analyzed by Northern blot and primer extension analysis. Terminally redundant R9 constructs containing the sequences of adw, ayw, MT5/6, MT5, MT6, and FH were transfected into HuH-7 cells. RNAs were purified and analyzed by Northern blot hybridization. Two species of HBV RNA, 3.5 and 2.4/2.1 kb, were present in all transfections (Fig. 5). In Fig. 5, densitometric quantitation showed a 2.4-fold increase of the MT5/6 3.5-kb RNA com-

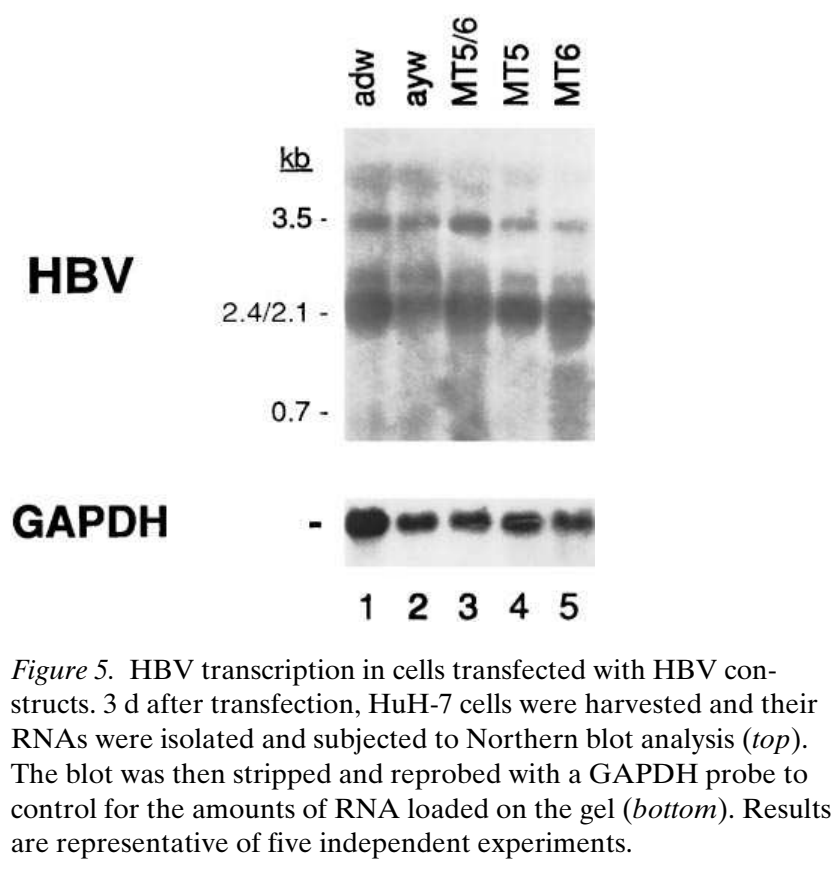

pared to the adw wild type when corrected for GAPDH analysis. The densitometric reading of 3.5-kb RNA for adw was 510 (arbitrary units) and for MT5/6 was 978; the densitometric reading of GAPDH for adw was 2335 and for MT5/6 was 1842. There was no significant difference in the 2.1- and 2.4-kb transcripts (Fig. 5). Although this twofold difference in $3.5-\mathrm{kb}$ RNA level between WT adw and MT5/6 appeared small, it was confirmed by four other independent experiments. To study the effect of MT5/6 on pregenomic RNA transcription directly, primer extension analysis was performed. Primer extension of total viral RNA revealed three extension products: the shortest, representing the pregenomic RNA, and the two slightly longer species, representing the precore RNAs. The

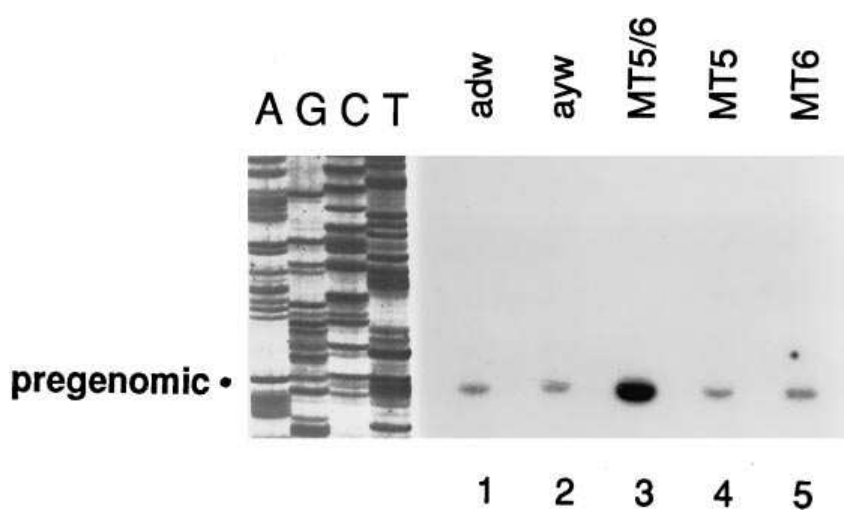

Figure 4. Primer extension analysis of encapsidated RNA. $3 \mathrm{~d}$ after transfection, core-associated RNA was purified and subjected to primer extension analysis. Primer extension products were electrophoresed on an $8 \%$ polyacrylamide-urea gel and autoradiography using an intensifying screen with exposure time of $24 \mathrm{~h}$ at $-80^{\circ} \mathrm{C}$ was performed. A sequencing reaction using the same primer on the adw strain is shown on the left (lanes marked $A, G, C, T$ ). Lanes are as indicated. Results are representative of four independent experiments.

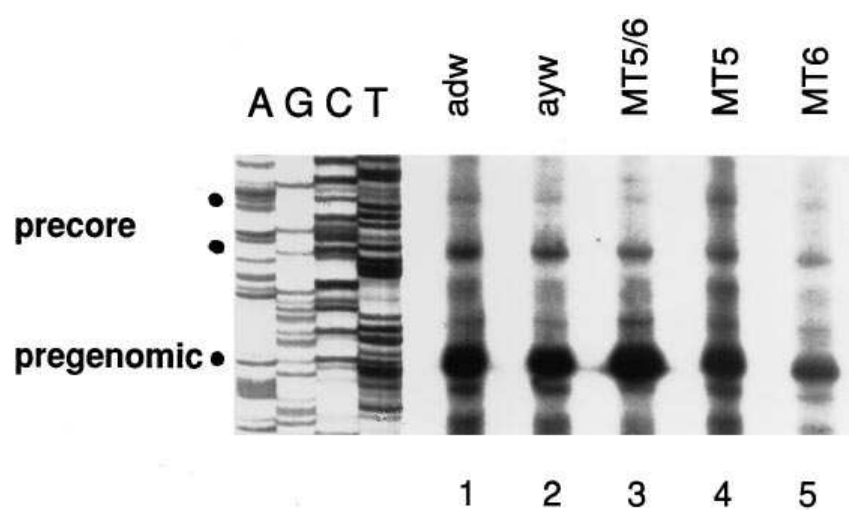

Figure 6. Primer extension analysis of total RNA. $3 \mathrm{~d}$ after transfection, $3 \mu \mathrm{g}$ of total RNA (1/50 of total RNA prepared from one $10-\mathrm{cm}$ dish) was subjected to primer extension analysis as described. Primer extension products were electrophoresed on an $8 \%$ polyacrylamideurea gel and autoradiography using an intensifying screen with exposure time of $24 \mathrm{~h}$ at $-80^{\circ} \mathrm{C}$ was performed. A sequencing reaction using the same primer on the adw strain is shown on the left (lanes marked $A, G, C, T)$. Lanes are as indicated. Results are representative of four independent experiments. 


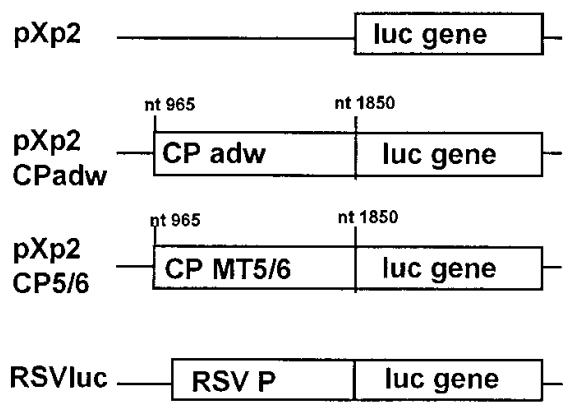

RSVluc \begin{tabular}{|l|l|}
\hline RSV $P$ & luc gene \\
\hline
\end{tabular}

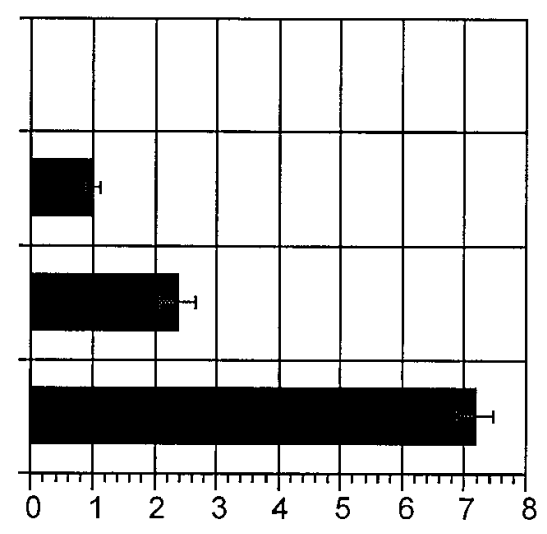

luciferase activity ( $10^{6}$ arbitrary light units)
Figure 7. Effects of MT5/6 on core promotor activity. Promotor constructs containing no (negative control), adw core promotor, MT5/6 core promotor, or RSV promotor (positive control) driving the luciferase gene were transfected into HepG2 cells. $48 \mathrm{~h}$ after transfection, luciferase activities were measured as described. Results are representative of four independent experiments. pregenomic RNA appeared to be the most abundant transcript (Fig. 6). The construct containing MT5/6 displayed an approximately twofold increase $(1.96 \pm 0.45$, from four experiments) of the pregenomic RNA transcript compared to the wild-type strains or the constructs with individual mutation 5 or 6 (a typical experiment is shown in Fig. 6). The level of precore RNA transcripts was not influenced by MT5/6, compared with the wild-type.

To confirm the minor effect of MT5/6 on core promotor activity, we inserted the wild-type and mutant (MT5/6) core promotors into the promotorless, luciferase gene plasmid $\mathrm{pXp} 2$. These constructs were transfected into HepG2 cells and luciferase expression was analyzed. Fig. 7 demonstrated that the core promotor mutation MT5/6 led to a 2.3-fold increase in the expression of the reporter gene.

In addition to regulating pregenomic RNA transcription, the HBV core promotor also directs transcription of precore RNAs. The precore RNAs are translated into the precore protein, which is posttranslationally processed and secreted as hepatitis B virus e antigen ( $\mathrm{HBe} \mathrm{Ag})$. As shown, primer extension analysis of total RNA did not reveal any significant difference in precore RNA transcription. To extend this observation, we studied the effect of MT5/6 on HBeAg synthesis.

Table I. HBV Protein Secretion in Medium of Cells Transfected with HBV Constructs

\begin{tabular}{lccr}
\hline Construct & hGH & HBsAg & HBeAg \\
\hline & $n g / m l$ & $S / N$ & $S / N$ \\
adw & 6.0 & 27.7 & 9.2 \\
ayw & 6.2 & 23.9 & 10.0 \\
MT5/6 & 5.9 & 22.6 & 11.2 \\
MT5 & 7.1 & 24.1 & 9.0 \\
MT6 & 6.5 & 25.0 & 9.6 \\
FH & 5.0 & 15.6 & 1.3 \\
\hline
\end{tabular}

Huh-7 cells were maintained and transfected with various constructs. Transfection efficiency was monitored by cotransfection with pTKGH and subsequent analysis of secreted $\mathrm{hGH}$ by radioimmunoassay. On day 3 , culture media were harvested for detection of hGH, HBsAg, and $\mathrm{HBeAg}$. Average values from $\mathrm{hGH}, \mathrm{HBsAg}$, and $\mathrm{HBeAg}$ radioimmunoassays are shown (for $\mathrm{HBsAg}$ and $\mathrm{HBeAg}$ a signal to noise ratio $[\mathrm{S} / \mathrm{N}]$ of $>2$ is considered positive). Results are representative of four independent experiments.
After transfection of HuH-7 cells with various wild-type and mutant constructs, secreted $\mathrm{HBeAg}$ and hGH (as control) were analyzed in the medium of the transfected cells. As shown in Table I, the identified core promotor mutations had no effect on the $\mathrm{HBeAg}$ synthesis. In contrast, the construct containing the fulminant strain led to the well-described absence of HBeAg synthesis (Table I). HBsAg production was not affected to any significant extent by any of the mutations (Table I).

MT5/6-induced enhanced replication is independent of $H B X$. Since the core promotor overlaps with the HBX gene, MT5/6 also affects the HBX gene. MT5/6 in the HBX ORF resulted in two amino acid substitutions in the HBX protein; valine is replaced by isoleucine in position 132 and phenylalanine is changed to tyrosine in position 133 . Since the biological function of HBX is not completely understood, it is difficult to assess the true effect of MT5/6 on HBX function. The only established function of HBX is its ability to transactivate transcription of a variety of cellular and viral genes (20-22). To examine the effect of MT5/6 on HBX transactivation, wild-type

pGEM7

HBXadw

HBXMT5/6

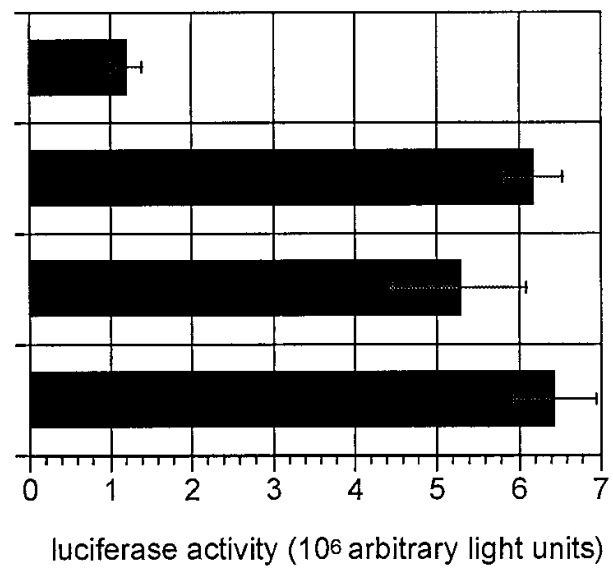

Figure 8. Transactivation of HBX mutants. A luciferase reporter construct directed by the RSV promotor was cotransfected with HBX wild-type (adw), MT5/6, and FH expression contructs into HepG2. The expression vector pGEM7 without HBX served as a negative control. Reporter activity was analyzed $48 \mathrm{~h}$ after transfection as described. The results are representative of four independent experiments. 
and mutant HBX constructs were compared in their ability to transactivate the luciferase reporter gene. After cotransfection of the HBX expression and reporter constructs into HepG2 cells, no appreciable difference in transactivation was detected between the wild type and mutants (Fig. 8).

To investigate whether the amino acid changes of the HBX protein are important for MT5/6-induced enhanced replication, MT5/6 was introduced into the second copy of the HBX coding sequence at the $3^{\prime}$ end of the terminal-redundant construct adwR9 (Fig. 2). In this position MT5/6 did not show the phenotype of enhanced viral replication (data not shown). To further elucidate the role of mutated HBXs in MT5/6-induced enhanced replication, the $\mathrm{HBX}$ open reading frame was inactivated in the replication-competent construct containing MT5/6 at its $5^{\prime}$ end (Fig. $9 A$ ). HBX contains three conserved, inframe start codons, each of which has been proposed to encode functionally related gene products $(23,24)$. Since the replication-competent R9 construct begins at nt 1417, it contains only the two translational initiation sites for the middle $(\mathrm{p} 8)$ and small (p6.6) HBX at its 5' end. Inactivation of these reading frames was obtained by introduction of a stop codon and a mutated start codon for p8 (HBX.2) and p6.6 (HBX.3) as shown in Fig. $9 A$. Analysis of replicative intermediates of these constructs transfected into $\mathrm{HuH}-7$ cells demonstrated that MT5/6-induced enhanced replication was independent of HBX inactivation (Fig. $9 B$ ).

$M T 5 / 6$ increases viral encapsidation independent of pregenomic RNA transcription level. The following experiments were designed to further characterize the mechanism by which the mutation(s) affect HBV encapsidation. Encapsidation of the HBV pregenomic RNA is thought to require the $\epsilon$ encapsidation signal on the pregenomic RNA, the core protein, and the polymerase protein $(5,6,19)$. Since MT5/6 appears to be outside these three elements, it likely affects viral encapsidation in a novel manner. It is not known, whether other components, e.g., other viral or cellular factors, are involved in the encapsidation process. By acting in cis and affecting the core promotor function with a twofold increase in pregenomic RNA transcription level, MT5/6 could cause a modest increase in viral encapsidation. However, this effect on pregenomic RNA transcription (twofold) was much smaller than the observed effect on encapsidation ( $>10$-fold) of pregenomic RNA by MT5/6. Therefore, mechanisms other than increased level of transcription of pregenomic RNA must be present. A possible effect of MT5/6 on additional yet unidentified encapsidation sequences could be the reason for more efficient encapsidation. However, this possibility is highly unlikely because mutations introduced only in the $5^{\prime}$ terminal redundant region of the $\mathrm{R} 9$ construct should not be present in the pregenomic RNA transcript (see Fig. 2).

To address this issue further, we applied a previously described encapsidation system (14). In this system a plasmid expressing a chimeric RNA (HBV $\epsilon$-lacZ) bearing the HBV $\epsilon$ encapsidation sequence fused to lac $Z$ is cotransfected with a plasmid expressing core and polymerase (pCMV-CP). The chimeric RNA was shown to encapsidate into core particles (14). This system would permit us to discern a direct cis effect of MT5/6 on pregenomic RNA transcription and encapsidation, or a transcript level-independent trans effect on encapsidation induced by MT5/6. To study the cis effect, we cotransfected the core promotor constructs pCPadwlacZ and pCPMT5/6lacZ together with pCMV-CP. MT5/6 resulted in increased encapsi-
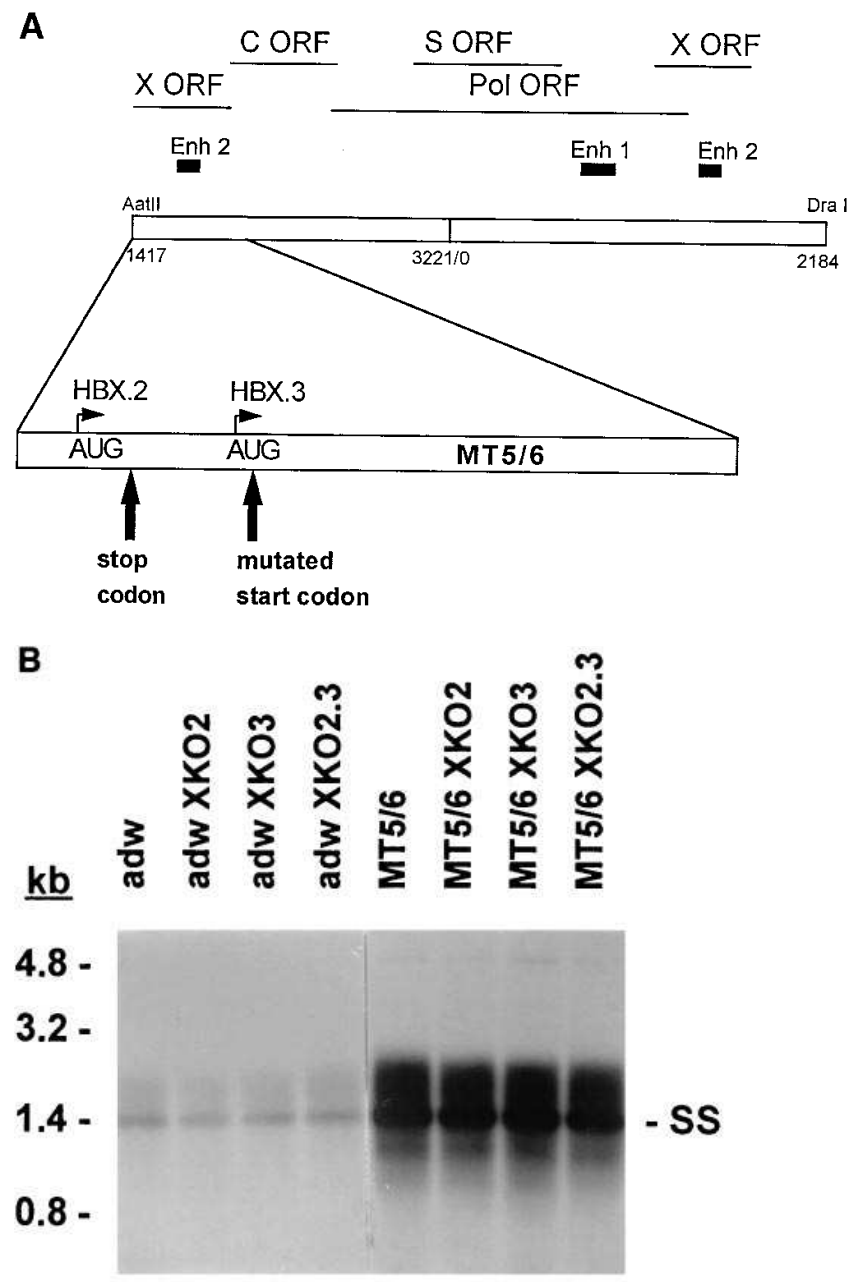

\section{$\begin{array}{llllllll}1 & 2 & 3 & 4 & 5 & 6 & 7 & 8\end{array}$}

Figure 9. Effect of HBX inactivation on MT5/6-induced enhanced replication. HBX translation was inactivated by introduction of a stop codon shortly after the start site for the second HBX translation product and by mutating the start codon for the third HBX translation product as illustrated in $(A)$. After transfection of the constructs into HuH-7 cells HBV replication was analyzed by Southern blot of core-associated replicative intermediates $(B)$. Lane content as indicated: XKO2.3, constructs with knockout of second and third HBX gene expression product as indicated in $(A)$. SS, single-stranded HBV-DNA. Results are representative of four independent experiments.

dation of HBV $\epsilon$-lacZ RNA by two to three times $(2.4 \pm 0.5$, from four experiments; Fig. $10 C$, lanes 7 and 8 ). The enhanced encapsidation was due to increased transcription of the chimeric RNA (Fig. $10 C$, lanes 1 and 2), which is consistent with our previous findings. Next, we provided the mutation(s) in trans by cotransfecting the wild-type construct pCPadwlacZ together with either adwR9 or MT5/6R9. In this experiment, MT5/6 led to a dramatic increase of encapsidation of HBV $\epsilon$ lacZ RNA (Fig. $10 C$, lanes 9 and 10) without affecting the transcription of HBV $\epsilon$-lacZ RNA (Fig. $10 C$, lanes 3 and 4). This blot was overexposed to reveal the weak signals of lanes 7 and 8 . In a lighter exposure, the significant differences between lanes 9 and 10 as well as between 11 and 12 were much 


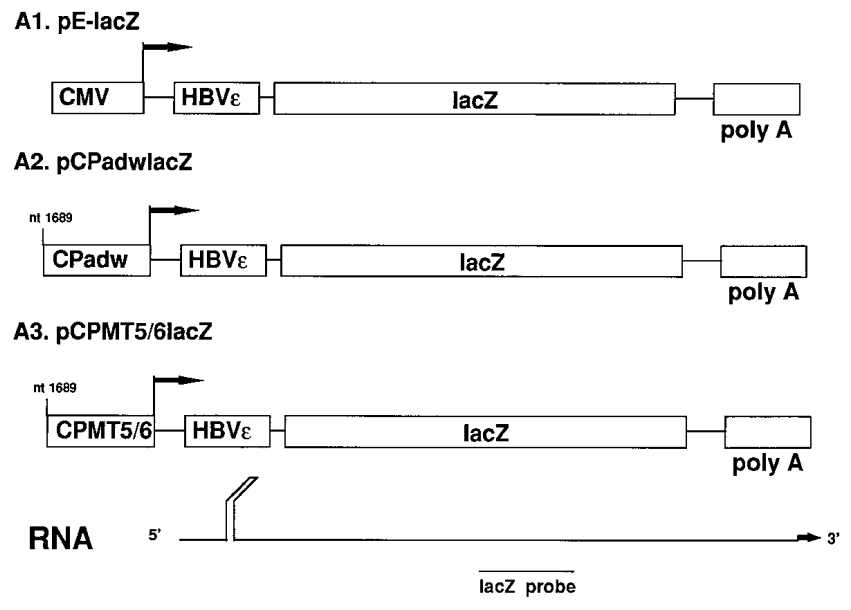

C
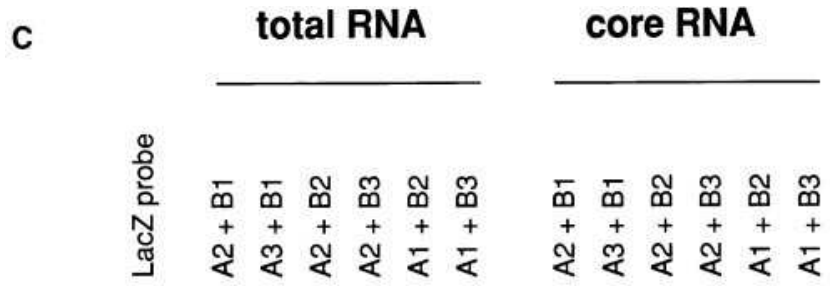

475 425 -

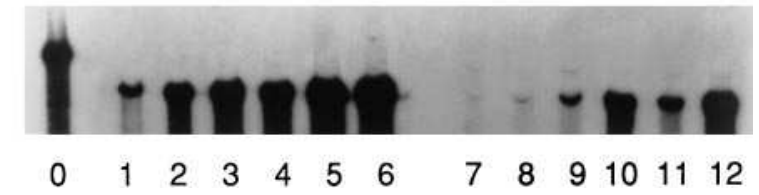

more evident. Densitometric quantification revealed a $>10$-fold difference $(10.9 \pm 2.1$, from four experiments) between the wild-type and mutant signal intensities. A similar effect was also evident when a heterologous CMV promotor construct pE-lacZ was used instead of pCPadwLacZ (Fig. $10 C$, lanes 11 and 12$)$. In the $\mathrm{pE}$-lacZ construct, the core promotor was replaced with the CMV promotor to drive the chimeric RNA. This experiment further supports the notion, that a major effect of MT5/6 on encapsidation is independent of the level of transcription.

\section{Discussion}

In our previous study, we demonstrated an HBV strain associated with an outbreak of fulminant hepatitis B exhibiting the phenotype of enhanced replication in tissue culture. We demonstrated that the increased level of core-associated HBV pregenomic RNA and replicative intermediates was associated with enhanced virion production in the medium (10). In this study, we defined the mutations of the FH strain responsible for the enhanced replication to the overlapping region of core promotor and distal HBX gene. The combined mutations of $\mathrm{C}$ to $\mathrm{T}$ at nucleotide position 1768 and $\mathrm{T}$ to $\mathrm{A}$ at nucleotide position 1770 were sufficient to induce the enhanced replication phenotype observed with the FH strain (Fig. 3). Similar to the FH strain, these mutations caused enhanced replication predominantly by an increased encapsidation of pregenomic RNA (Fig. 4). These two mutations were present in all the clones iso-
B1. pCMV-CP

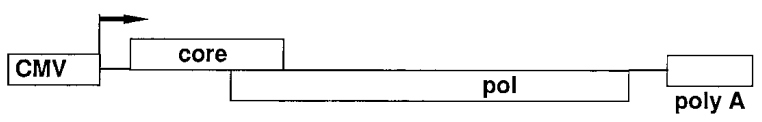

B2. adwR9

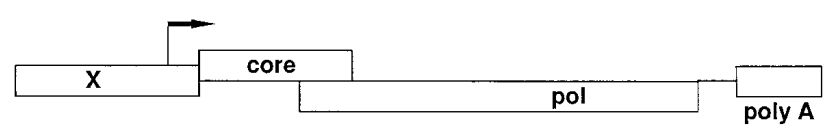

B3. MT5/6R9

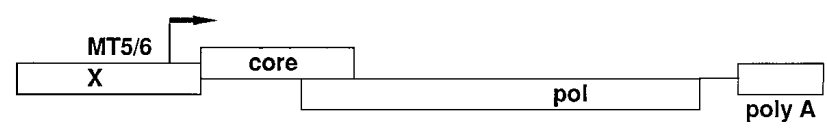

Figure 10. Characterization of the effects of MT5/6 on encapsidation. (A) Structural organization of various HBVlacZ constructs. Construct A3 contains the MT5/6 in cis. (B) Structural organization of the packaging constructs. Construct B3 contains MT5/6 in trans. $(C)$ Ribonuclease protection analysis of HBVLacZ RNA. $3 \mathrm{~d}$ after transfection, total and core-associated RNAs were purified and 1/10 of total RNA and all core RNA were subjected to ribonuclease protection analysis as described. Riboprobe (lane 0 ) and protected fragments were electrophoresed (lanes 1-12) on an $8 \%$ polyacrylamide-urea gel and autoradiography using an intensifying screen with an exposure time of $48 \mathrm{~h}$ at $-80^{\circ} \mathrm{C}$ was performed. The nucleotide length of riboprobe $(475 \mathrm{nt})$ and protected fragment $(425 \mathrm{nt})$ are indicated on the left side. Lane 0 , riboprobe; lanes 1-6, total RNA, representing transcription of HBVlacZ RNA; lanes 712, core-associated RNA, reflecting encapsidation of HBVlacZ RNA. The results are representative of four independent experiments.

lated from the five patients with fulminant hepatitis as well as the source patient who transmitted the virus (9). This observation indicated that these two mutations were not a result of PCR or cloning artifacts. Although other mutations in this viral strain may have other phenotypic effects, our data suggest that these two mutations resulting in enhanced replication are likely a unique feature of this naturally occurring HBV mutant.

Previous studies suggested that HBV replication is higher in postconfluent cells (15). It is possible that MT5/6 relieves the virus of this dependence to result in an overall higher replication. We have analyzed viral replication and observed enhanced replication of the mutant strain at various time points in culture. Thus far, mutations leading to enhanced replication have been described only in avian hepadnavirus. In duck hepatitis B virus, an amino acid change in the large envelope protein has been shown to enhance viral replication by increasing the cellular pool of ccc DNA (25).

Enhanced replication of the FH strain was the result of enhanced encapsidation of pregenomic RNA. Enhanced encapsidation was caused by two mechanisms: First, MT5/6 resulted in a two- to threefold increase in pregenomic RNA transcription, leading to a similar increase in viral encapsidation (Fig. 10). Second, MT5/6 led to a transcript-independent increase in encapsidation efficiency ( $>10$-fold) by a yet unidentified mechanism (Fig. 10).

Increased transcription of pregenomic RNA was the result of altered core promotor function induced by the mutations. The core promotor regulates the transcription of the prege- 
nomic RNA which is translated into the core protein and serves as the template for reverse transcription during viral replication (1). Primer extension analysis and Northern blot analyses of total RNA showed an approximately twofold increase in transcription of the pregenomic RNA in MT5/6 as compared with the wild-type adw strain (Figs. 5 and 6). Using a luciferase reporter, we observed a similar effect (2.3-fold increase) of the mutations on core promotor activities (Fig. 7). In addition to directing pregenomic RNA transcription, the core promotor regulates transcription of the precore RNAs which are translated into $\mathrm{HBeAg}(26,27)$. Primer extension analysis of total RNA and analysis of HBeAg synthesis demonstrated that MT5/6 had no significant effect on transcription and expression of the precore gene. Recently, it was suggested that the precore expression product ( $\mathrm{HBeAg}$ precursor protein) can inhibit HBV DNA synthesis by interfering with viral encapsidation (28). Since precore gene expression was not affected by MT5/6, the effect of MT5/6 on viral encapsidation via this mechanism seems to be unlikely.

In addition to increased transcription of pregenomic RNA, encapsidation was enhanced by a mechanism apparently independent of the level of pregenomic RNA transcription (Fig. 10). Encapsidation of pregenomic RNA has been shown to require the $\epsilon$ encapsidation signal present in the pregenomic RNA, the core, and the polymerase $(5,6,14,19)$. Our experiments suggest that the sequence in the core promotor-HBX gene may have a yet unidentified role in the regulation of the encapsidation process. Several mechanistic pathways are possible to explain the observed transcript-independent effect of MT5/6 on viral encapsidation.

First, encapsidation may be increased by a viral trans-acting factor. Such a trans-acting factor could be the HBX protein. Various forms of HBX protein, based on translation initiation at three different AUG start codons within the HBX open reading frame, have been postulated to exist $(23,24)$. The biological importance of the HBX protein(s) in viral life cycle remains largely unknown. Full-length HBX has been shown to be dispensable for HBV replication in tissue culture (29) but necessary for establishment of woodchuck hepatitis virus infection in vivo $(30,31)$. In transgenic mice, high level viral replication could be achieved only with a terminally redundant HBV transgene containing a duplicate HBX gene at its $5^{\prime}$ end (32). HBV transgenes without the HBX coding region in the $5^{\prime}$ end yielded no or low level HBV replication in mice (32). Although these transgenes contain an additional copy of the entire HBX gene at their $3^{\prime}$ end, this copy is apparantly not sufficient to direct high level viral replication (32). In our system, MT5/6 placed in the $3^{\prime}$ terminal redundant region of the R9 construct had no effect on viral replication or encapsidation. Inactivation of HBX translation by oligonucleotide-directed mutagenesis did not abolish the MT5/6-induced enhanced replication (Fig. 9). In addition, introduction of MT5/6 in a HBX expression construct did not alter the transactivation of a luciferase reporter gene (Fig. 8). These findings essentially exclude an important role for the HBX gene product in mediating MT5/6-induced enhanced replication. Alternatively, transcripts or proteins of an antisense open reading frame (ORF) or spliced sequences such as those proposed earlier $(30,33)$ may mediate a trans effect on viral encapsidation. However, inactivation of an identified antisense open reading frame by inserting a frame-shift mutation did not abolish MT5/6 induced enhanced replication (data not shown).
Second, enhanced encapsidation could be the result of a cotranscriptional effect on core and polymerase expression induced by MT5/6. Such a cotranscriptional effect could result in a more efficient core or polymerase expression leading to an increased assembly of the encapsidation components. Translation of the RT-Pol appeared to occur through a novel ribosomal scanning mechanism $(34,35)$, probably depending on dynamic interaction of cellular initiation and elongation factors bound to the pregenomic transcript. Since the RT-Pol is probably the limiting factor for encapsidation, it is conceivable that MT5/6 alters the binding complex of these cellular factors during transcription leading to increased RT-Pol translation. MT5/6 may also act post- or cotranscriptionally by interacting with cellular factors required for RT-Pol activity and the subsequent encapsidation process. A cellular factor, Hsp90, has been recently identified to interact with the replication complex (36). Further experiments are under way to identify possible viral and/or cellular elements involved in MT5/6-induced enhanced encapsidation.

Regardless of the mechanism, our study demonstrates an important role for the core promotor sequence in HBV encapsidation and replication. Our current data, however, do not distinguish between whether MT5/6 exerts a positive or negative effect on this yet unknown element which could have either an activating or repressing role in encapsidation and replication. Further studies are neccessary to clarify whether this finding is unique to this $\mathrm{FH}$ strain or it is important for HBV replication in general. Mutations in the core promotor region have been detected in patients with fulminant hepatitis B in Japan (37) and Europe (38) but less frequently in the U.S. (39). Our identified C-to-T mutation at nucleotide position 1768 and T-to-A mutation at nucleotide position 1770 were present in one of the Japanese strains (37). Core promotor mutations have also been described in HBV carriers and patients with chronic hepatitis (40). These studies led to the conclusion that core promotor mutations may influence the biological behavior of the virus, leading to a different clinical course. The authors of these studies speculated that transcription of the precore mRNA might be decreased as a result of these mutations leading to diminished HBeAg synthesis. Furthermore, they suggested that both the precore stop codon and core promotor mutation(s) are functionally equivalent resulting in a common phenotype of HBeAg deficiency. However, no functional studies have been performed to show that these mutations indeed have an effect on viral protein expression. Our study showed that $\mathrm{HBeAg}$ production was not affected by the specific core promotor mutations 5 and 6 (Table I). Functional studies of the core promotor sequences of other viral strains associated with fulminant hepatitis are necessary to elucidate the effect of core promotor mutations on viral replication. Furthermore, we have no direct evidence that the enhanced replication of the FH strain contributes to the development of fulminant hepatitis. Studies in an animal model, such as in woodchuck, would be crucial in demonstrating the biological significance of these mutations in the pathogenesis of HBV infection.

\section{Acknowledgments}

The authors thank Jiakang Huang and Margaret Koziel (GI Unit, Massachusetts General Hospital, Boston, MA) and Jesse Summers (University of New Mexico, Albuquerque, NM) for helpful discus- 
sions, and Don Ganem (University of California, San Francisco, CA) for generously providing the constructs placZ, pE-LacZ, and pCMV-CP.

This work was supported by a postdoctoral fellowship grant from the Deutsche Forschungsgemeinschaft (Ba 1417/1-1) to T.F. Baumert, and grants from the National Institutes of Health to T.F. Baumert (VF-DK-14361) and T.J. Liang (DK-01952, CA-54524).

\section{References}

1. Ganem, D., and H.E. Varmus. 1987. The molecular biology of the hepatitis B virus. Annu. Rev. Biochem. 56:651-693.

2. Tiollais, P., C. Pourcel, and A. Dejean. 1985. The hepatitis B virus. Nature (Lond.). 317:489-495.

3. Summers, J., and W.S. Mason. 1982. Replication of the genome of a hepatitis B-like virus by reverse transcription of an RNA intermediate. Cell. 29: 403-415

4. Will, H., W. Reiser, T. Weimer, E. Pfaff, M. Buescher, R. Sprengel, R. Cattaneo, and H. Schaller. 1987. Replication strategy of human hepatitis B virus. J. Virol. 61:904-911.

5. Hirsch, R.C., J.E. Lavine, L.-J. Chang, H.E. Varmus, and D. Ganem. 1990. Polymerase gene products of hepatitis B viruses are required for genomic RNA packaging as well as for reverse transcription. Nature (Lond.). 344:552-555.

6. Junker-Niepmann, M., R. Bartenschlager, and H. Schaller. 1990. A short cis-acting sequence is required for hepatitis B virus pregenome encapsidation and sufficient for packaging of foreign RNA. EMBO (Eur. Mol. Biol. Organ.) J. 9:3389-3396.

7. Nassal, M., M. Junker-Niepmann, and H. Schaller. 1990. Translational inactivation of RNA function: discrimination against a subset of genomic transcripts during HBV nucleocapsid assembly. Cell. 63:1357-1363.

8. Pollack, J.R., and D. Ganem. 1994. Site-specific RNA binding by a hepatitis B virus reverse transcriptase initiates two distinct reactions: RNA packaging and DNA synthesis. J. Virol. 68:5579-5587.

9. Liang, T.J., H. Hasegawa, N. Rimon, J.R. Wands, and E. Ben-Porath. 1991. A hepatitis B virus mutant associated with an epidemic of fulminant hepatitis. N. Engl. J. Med. 324:1705-1709.

10. Hasegawa, K., J. Huang, S.A. Rogers, H.E. Blum, and T.J. Liang. 1994. Enhanced replication of a hepatitis B virus mutant associated with an epidemic of fulminant hepatitis. J. Virol. 68:1651-1659.

11. Chen, H.-S., M.C. Kew, W.E. Hornbuckle, B.C. Tennant, P.J. Cote, J.L. Gerin, R.H. Purcell, and R.H. Miller. 1992. The precore gene of the woodchuck hepatitis virus genome is not essential for viral replication in the natural host. $J$. Virol. 66:5682-5684.

12. DeWet, J.R., K.V. Wood, M. DeLuca, D.R. Helinski, and S. Subramani. 1987. Firefly luciferase gene: structure and expression in mammalian cells. Mol. Cell. Biol. 7:725-737.

13. Nordeen, S.K. 1988. Luciferase reporter gene vectors for analysis of promoters and enhancers. Biotechniques. 6:454-457.

14. Pollack, J.R., and D. Ganem. 1993. An RNA stem-loop structure directs hepatitis B virus genomic RNA encapsidation. J. Virol. 67:3254-3263.

15. Sureau, C., J.-L. Romet-Lemonne, J.I. Mullins, and M. Essex. 1986. Production of hepatitis B virus by a differentiated human hepatoma cell line after transfection with cloned circular DNA. Cell. 47:37-47.

16. Chomczynski, P.N., and N. Sacchi. 1987. Single-step method of RNA isolation by acid guanidinium thiocyanate-phenol-chloroform extraction. Anal. Biochem. 162:156-159.

17. Blum, H.E., E. Galun, T.J. Liang, F. von Weizsäcker, and J.R. Wands. 1991. Naturally occurring missense mutation in the polymerase gene terminating hepatitis B virus replication. J. Virol. 65:1836-1842.

18. Ausubel, F.M., R. Brent, R.E. Kingston, D.D. Moore, J.G. Seidman, J.A. Smith, and K. Struhl. 1991. Current Protocols in Molecular Biology. John Wiley \& Sons, New York.

19. Lavine, J., R. Hirsch, and D. Ganem. 1989. A system for studying the se- lective encapsidation of hepadnavirus RNA. J. Virol. 63:4257-4263.

20. Colgrove, R., G. Simon, and D. Ganem. 1989. Transcriptional activation of homologous and heterologous genes by the hepatitis $\mathrm{B}$ virus $\mathrm{X}$ gene product in cells permissive for viral replication. J. Virol. 63:4109-4026.

21. Seto, E., P.J. Mitchell, and T.S.B. Yen. 1990. Transactivation by the hepatitis $\mathrm{B}$ virus $\mathrm{X}$ protein depends on AP-2 and other transcription factors. Nature (Lond.). 344:72-74

22. Maguire, H.F., J.P. Hoeffler, and A. Siddiqui. 1991. HBV X protein alters the DNA binding specificity of CREB and ATF-2 by protein-proteininteractions. Science (Wash. DC). 252:842-844.

23. Kwee, L., R. Lucito, B. Aufiero, and R.J. Schneider. 1992. Alternate translation initiation on hepatitis B virus $\mathrm{X}$ mRNA produces multiple polypeptides that differentially transactivate class II and III promotors. J. Virol. 66 : 4382-4389.

24. Zheng, Y.-W., J. Riegler, J. Wu, and T.S.B. Yen. 1994. Novel short transcripts of hepatitis B virus X gene derived from intragenic promotor. J. Biol. Chem. 269:22593-22598.

25. Lenhoff, R.J., and J. Summers. 1994. Coordinate regulation of replication and virus assembly by the large envelope protein of an avian hepadnavirus. J. Virol. 68:4565-4571.

26. Yaginuma, K. and K. Koike. 1989. Identification of a promoter region for 3.6-kilobase mRNA of hepatitis B virus and specific cellular binding protein. J. Virol. 63:2914-2920.

27. Yuh, C.-H., Y.-L. Chang, and L.-P. Ting. 1992. Transcriptional regulation of precore and pregenomic RNAs of hepatitis B virus. J. Virol. 66:40734084 .

28. Lamberts, C., M. Nassal, I. Velhagen, H. Zentgraf, and C.H. Schroeder. 1993. Precore-mediated inhibition of hepatitis B virus progeny DNA synthesis. J. Virol. 67:3756-3762.

29. Blum, H.E., Z.-S. Zhang, E. Galun, F. von Weizsäcker, B. Garner, T.J. Liang, and J.R. Wands. 1992. Hepatitis B virus X protein is not central to the viral life cycle in vitro. J. Virol. 66:1223-1227.

30. Chen, H.-S., S. Kaneko, R. Girones, R.W. Anderson, W.E. Hornbuckle, B.C. Tennant, P.J. Cote, J.L. Gerin, R.H. Purcell, and R.H. Miller. 1993. The woodchuck hepatitis X gene is important for establishment of virus infection in woodchucks. J. Virol. 67:1218-1226.

31. Zoulim, F., J. Saputelli, and C. Seeger. 1994. Woodchuck hepatitis virus $\mathrm{X}$ protein is required for viral infection in vivo. J. Virol. 68:2026-2030.

32. Guidotti, L.G., B. Matzke, H. Schaller, and F.V. Chisari. 1995. Highlevel hepatitis B virus replication in transgenic mice. J. Virol. 69:6158-6169.

33. Miller, R.H., S. Kaneko, C.T. Chung, R. Girones, and R.H. Purcell. 1989. Compact organization of the hepatitis B virus genome. Hepatology. 9: 322-327.

34. Fouillot, N., S. Tlouzeau, J.-M. Rossignol, and O. Jean-Jean. 1993. Translation of the hepatitis B virus $\mathrm{P}$ gene by ribosomal scanning as an alternative to internal initiation. J. Virol. 67:4886-4895.

35. Chang, L.-J., D. Ganem, and H.E. Varmus. 1990. Mechanism of translation of the hepadnaviral polymerase (P) gene. Proc. Natl. Acad. Sci. USA. 87 $5158-5162$.

36. Hu, J., and C. Seeger. 1996 . Hsp90 is required for the activity of a hepatitis B virus reverse transcriptase. Proc. Natl. Acad. Sci. USA. 93:1060-1064.

37. Sato, S., K. Suzuki, Y. Akahane, K. Akamatsu, K. Akiyama, K. Yunomura, F. Tsuda, T. Tanaka, H. Okamoto, Y. Miyakawa, and M. Mayumi. 1995. Hepatitis B virus strains with mutations in the core promotor in patients with fulminant hepatitis. Ann. Intern. Med. 122:241-248.

38. Sterneck, M., S. Guenther, T. Sanantonia, L. Fischer, X. Rogiers, C.E. Broelsch, and H. Will. 1996. The complete nucleotide sequence of hepatitis B virus in 9 patients with fulminant hepatitis B infection. Hepatology. 24:300-306.

39. Laskus, T., J. Rakela, M.J. Nowicki, and D.H. Persing. 1995. Hepatitis B virus core promotor sequence analysis in fulminant and chronic hepatitis B. Gastroenterology. 109:1618-1623.

40. Okamoto, H., F. Tsuda, Y. Akahane, Y. Sugai, M. Yoshiba, K. Moriyama, T. Tanaka, Y. Miyakawa, and M. Mayumi. 1994. Hepatitis B virus with mutations in the core promotor for an e antigen-negative phenotype in carriers with antibody to e antigen. J. Virol. 68:8102-8110. 